\title{
Systematic review and meta-analysis of veterinary-related occupational exposures to hazards
}

https://10.1515/ovs-2020-0104

Received Nov 16, 2020; accepted Feb 15, 2021

\begin{abstract}
Understanding hazards within the veterinary profession is critical for developing strategies to ensure the health and safety of personnel in the work environment. This study was conducted to systematically review and synthesize data on reported risks within veterinary workplaces. A systematic review of published data on occupational hazards and associated risk factors were searched within three database platforms namely PubMed, Ebscohost, and Google scholar. To determine the proportion estimates of hazards and pooled odds ratio, two randomeffects meta-analysis were performed. For the biological, chemical and physical hazards, the pooled proportion estimates were 17\% (95\% CI: 15.0-19.0, p < 0.001), 7.0\% (95\% CI: $6.0-9.0 \%, \mathrm{p}<0.001)$ and 65.0\% (95\% CI: 39.0-91.0\%, $\mathrm{p}<$ $0.001)$ respectively. A pooled odds ratio indicated the risk of exposures to physical (OR=1.012, 95\% CI: 1.008-1.017, $\mathrm{p}<0.001)$ and biological hazards (OR=2.07, 95\% CI: 1.702.52, $\mathrm{p}<0.001$ ) increased when working or in contact with animals. The review has provided a better understanding of occupational health and safety status of veterinarians and gaps within the developing countries. This evidence
\end{abstract}

\footnotetext{
*Corresponding author: oluwawemimo ADEBOWALE, Department of Veterinary Public Health and Preventive Medicine, Federal University of Agriculture, Alabata, Abeokuta, Ogun State, Nigeria. mail: oluwawemimo1@yahoo.com Olubunmi Gabriel FASANMI, Department of Veterinary Laboratory Technology, Federal College of Animal Health and Production Technology, Oyo State, Nigeria.

Babafela AWOSILE, Department of Health Information and Performances, Health PEI Charlottetown, PE Canada Monsurat AFOLABI, Department of Veterinary Public Health and Preventive Medicine, Federal University of Agriculture, Alabata, Abeokuta, Ogun State, Nigeria

Folorunso Oludayo FASINA, ECTAD, Food and Agriculture Organization of the United Nations (FAO), Dar es Salaam, Tanzania Department of Veterinary Tropical Diseases, University of Pretoria, Pretoria, South Africa
}

calls for policy formulation and implementation to reduce the risks of exposures to all forms of occupational-related hazards in veterinary workplaces.

Keywords: Systematic Review, Meta-analysis, Occupational hazards, Veterinary Profession

\section{Introduction}

Occupational hazards are injuries or ailments resulting from the work one does or from the environment in which one works [1]. Occupational exposures to hazards contribute immensely to the burden of diseases globally [1]. Approximately $2.3-2.7$ million workers die from workrelated injuries or illnesses [2,3] and a total economic loss of about $\$ 2.99$ trillion annually are documented $[1,3]$.

The veterinary profession is comprised of a diverse group of individuals who interact with a wide variability of animal species under a working environment that creates exposure to injuries [4]. In the United States (US), the veterinary profession has been ranked as the fifth-highest industry for the incidence of non-fatal work related injuries. This is not far behind police and fire protection services, while the human health professionals were not in the top 20 [5]. Although, some reports showed exposure to work-related hazards are more common in the developed than developing countries [6], whether this is a true assessment is doubtful and indeterminate because most African countries may lack occupational health and safety standards, implementations, risks assessment, and adequate reporting protocols.

The reported processes of injuries in the profession included physical hazards such as needlestick injuries (NSI), animal bites, kicks, scratches, and crushing by equipment used for animal restraint, which imposed physical harm or hurt to individuals $[5,7]$. Other occupational threats include exposure

Ә Open Access. (C) 2020 Oluwawemimo Adebowale, published by De Gruyter. (G) BY This work is licensed under the Creative Commons Attribution 4.0 Public License. 
to biological (especially zoonoses), and chemical hazards (high doses of radiation and pesticides) which increase the risk of birth defects in offspring of female veterinarians [8]. Scientific data have also disclosed seroprevalence against different zoonoses is greater among veterinarians than in the general population, suggesting that veterinarians could act as sentinels to detect emergent diseases and propagators of infections [6]. Common zoonoses found primarily associated with health risks or illnesses among veterinary students and professionals are caused primarily by bacteria, parasites, viruses, and fungi [6].

Systematic reviews and meta-analyses of occupational health hazards in the veterinary profession are rare, and to date only one systematic review addressing this subject especially zoonoses in veterinary students has been published [6]. Therefore, this work aims to assess available pieces of evidence regarding exposures to zoonoses/biological, physical, and chemical threats among professionals and students in the veterinary work environment, and synthesize the associated risk factors.

\section{Methodology}

\subsection{Study Type and Search approach}

A systematic literature review and meta-analysis was conducted to identify scientific articles documenting hazards, investigate risk factors or practices and association with exposures. Preferred Reporting Items for Systematic Reviews and Meta-analyses Statement (PRISMA) was used to summarize the article selection process. To select important papers, systematic search within PubMed, Ebscohost and Google scholar database freely available to us was performed. The search strategy included the key terms "occupational hazards", "zoonoses", "veterinary students", "veterinarians”, "risk factors", which were combined with the Boolean operator "AND" "OR". Paper selections were based on information provided in the titles and/or abstracts.

\subsection{Eligibility criteria for the selection of relevant materials}

Only papers published in English were eligible, and no restrictions were placed on the location of studies, except time (2007 - 2017). The following inclusion criteria for selection were used: 1 ). Paper title and abstract addressed the questions of interest i.e. "occupational health hazards report on veterinary professionals and students 2). Papers should be cross-sectional observational studies, cohort, case-control studies relevant to research focus 3). Studies that provided association data either univariable or multivariable analyses were included. 4). Confirmed zoonoses reports using laboratory detection methods where available. Mining and filtering of articles were carried out based on set inclusion criteria, and paper abstracts that reported intervention studies, reviews, systematic reviews and meta-analysis, and conference proceedings were excluded. Two of the authors independently read and examined all titles and abstracts of papers. Duplicated studies were removed and approval from both authors was obtained for a paper to be included.

\subsection{Data extraction and analysis}

Data extracted onto Excel spreadsheet (Microsoft Office Package 2016; Microsoft Corporation, Redmond, USA) included: 1) year of publication, 2) Authors, 3) location of study, 4) study design, 5) study population, 6) identified hazards, 7) laboratory methods, 8) prevalence and, 9) associated risk factors. Initial descriptive statistics were carried out to summarize the various data retrieved. Then, random-effects meta-analysis was carried out to allow for any heterogeneity between studies. Two random-effects meta-analysis were done, firstly, to calculate the pooled (weighted) proportions with respective 95\% confidence intervals for the different types of occupational hazards (i.e. biological, physical, and chemical hazards) among veterinarians. Second meta-analysis was done to calculate the pooled (weighted) ratio measures (Odds ratio or relative risk) of risk factors associated with occupational hazards. The risk factor meta-analysis was only done for working or contact with animals as a risk factor for occupational hazards among veterinarians. This is premised on sufficient published articles reporting ratio measures for working or contact with animals compared with other reported risk factors. The pooled prevalence and associated study estimate, and pooled odds ratio and study estimates were presented using forest plots. The $I^{2}$ statistic (a measure of inconsistency) was used to assess the variation between studies due to heterogeneity. A value of $0 \%$ shows no observed heterogeneity; increasing values indicate increasing heterogeneity. The $I^{2}$ statistic with cutoff values $\leq 25 \%, 26-\leq 50 \%$, and $\geq 75 \%$ was considered as low, moderate, and substantial heterogeneity. Subgroup analysis was performed to account for potential sources 
of heterogeneity between studies. Statistical significance was set at $\mathrm{P}<0.05$ while statistical analysis was carried out using STATA SE/15.0 (College Station, Texas 77845 USA).

\section{RESULTS}

\subsection{Literature search outcome}

A total of 33 articles from 6 continents were retained for the review following paper filtering for eligibility Figure 1.

Rejections were mainly based on the unavailability of either the prevalence data or risk associations. Summary of the article selection process presented in Figure 2.

\subsection{Characteristics of included published articles}

Reviewed papers originated from Africa $(\mathrm{n}=2)$, Asia (n $=4)$, Australia/Oceania $(n=4)$, Europe $(n=12)$, North America $(\mathrm{n}=8)$, and South America $(\mathrm{n}=3)$. The target populations included: 1) Veterinarians $(57.6 \%, 95 \% \mathrm{CI}$ [40.8 - 72.3]), 2) Veterinary students $(9.1 \%, 95 \%$ CI [2.4 $24.3]), 3)$ Veterinarians and Veterinary students $(9.1 \%, 95 \%$
CI [2.4 - 24.3]), and 4) Veterinarians, veterinary students, para-veterinarians and livestock workers $(24.4 \%, 95 \%$ CI [12.6 - 41.3]). A total of 10 studies (30.3\%) employed serology , $5(15.1 \%)$ culture and molecular, 3(9.1\%) culture and serology, and 2 articles (6.1\%) each used molecular methods only, and culture, serology and molecular detection techniques respectively. The characteristics of the papers included in this review are further described in Table 1.

\subsection{Hazard exposure and risk factors}

\subsubsection{Physical hazards}

The most common physical hazard identified was needlestick injury (NSI), followed by cumulative traumatic injuries/disorders. High prevalence exposure to needlestick injuries (range: 65.0 - 79.5\%) among the veterinary practice was commonly reported $[4,5,9,7]$. Being a male (OR 2.8, 95\% CI 1.4-6.0, and working with poultry daily (OR 2.4, 95\% CI 1.1-6.2) were found to be significantly associated with NSI in a study conducted in Nigeria [7]. In a similar study, veterinarians working in small animal practice especially with dogs were

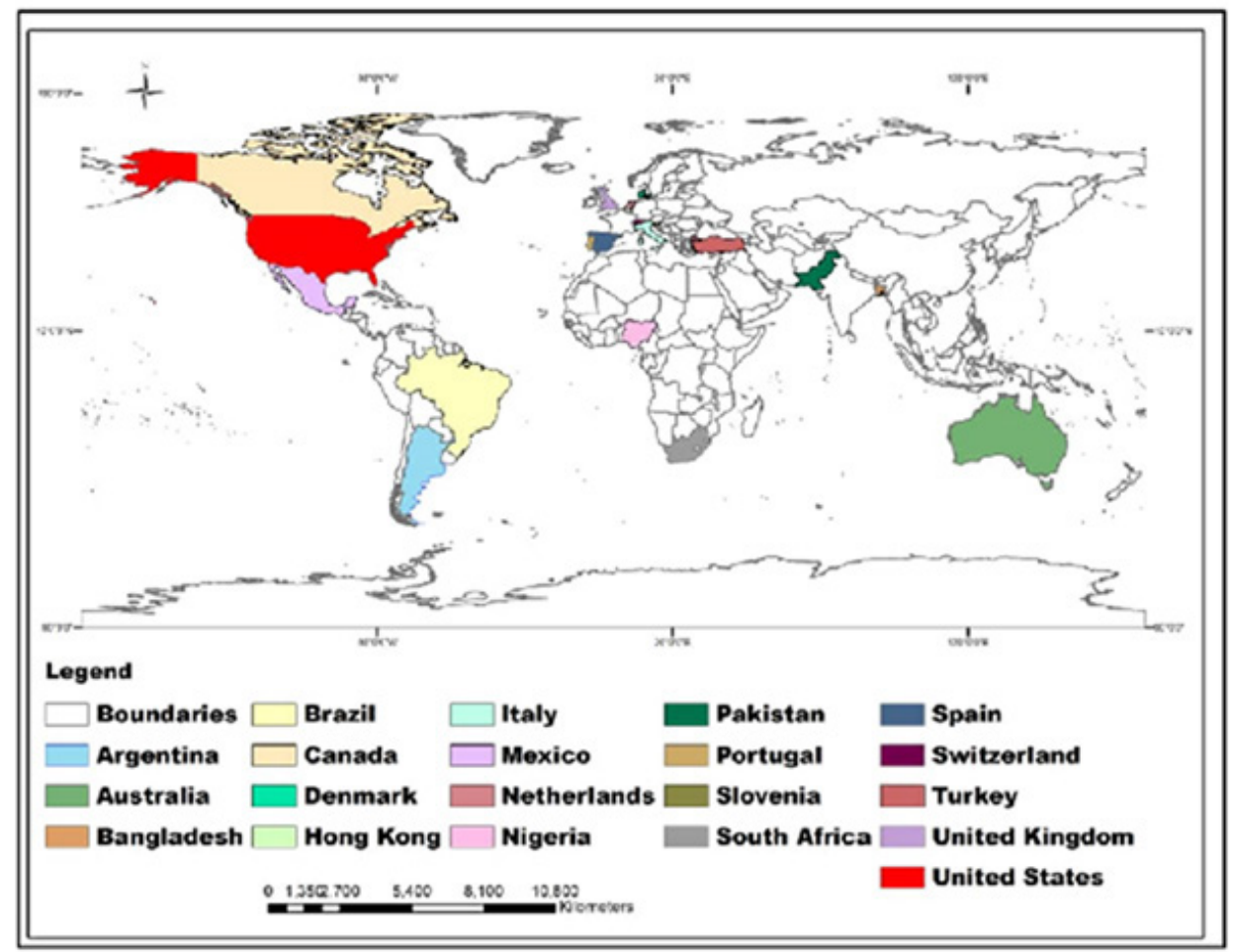

Figure1. Spatial distribution of countries from which information on occupational hazards and exposures among the veterinary profession were retrieved. 


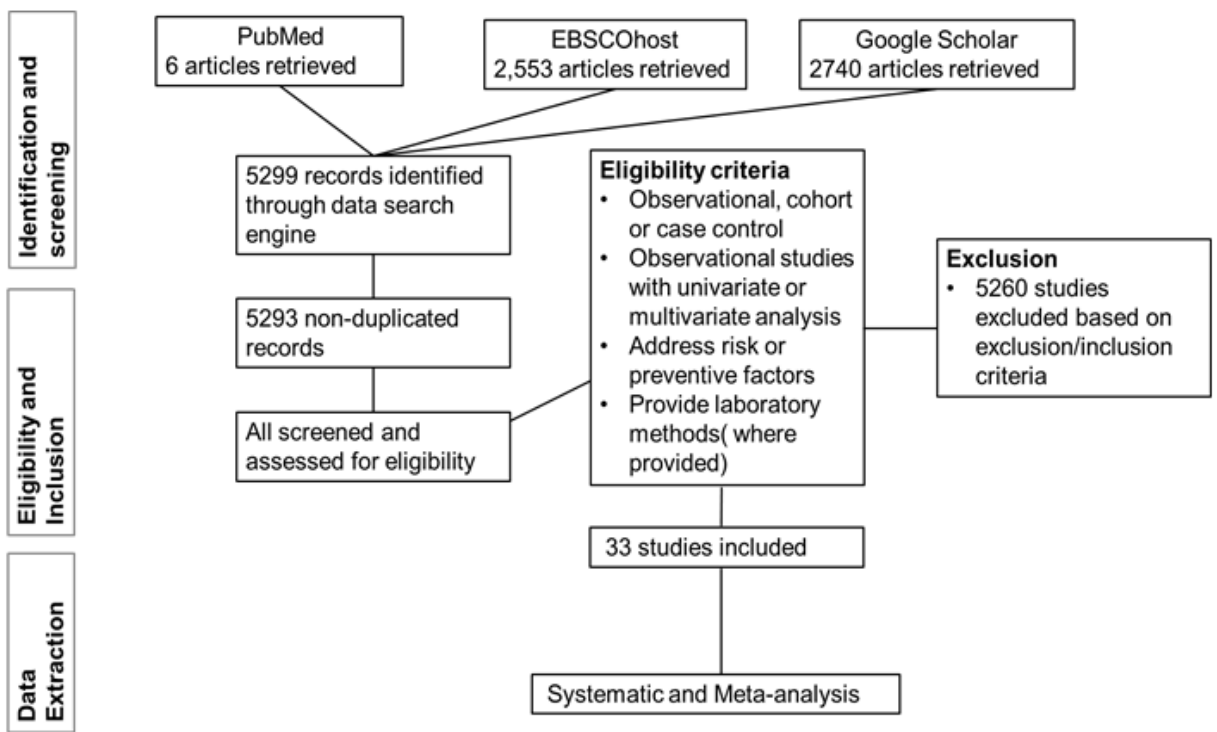

Figure 2. Flow chart of study selection for the systematic review and meta-analysis

Table 1 Summary of identified article characteristics according to year, authors, location, study design, and hazard types.

\begin{tabular}{|c|c|c|c|c|c|c|}
\hline $\mathrm{S} / \mathrm{N}$ & Year & Authors & Location & Study design & Hazard type & References \\
\hline 1. & $2012 a$ & Epp \& Waldner & Canada & Cross- sectional & Biological, Chemical, Physical & [4] \\
\hline 2. & 2016 & Fowler et al & USA & Cross-sectional & Biological, Chemical, Physical & [5] \\
\hline 3. & 2016 & Mshelbwala et al & Nigeria & Cross-sectional & Physical & [7] \\
\hline 4. & 2009 & Shirangi et al & Australia & Cross-sectional & Chemical & [8] \\
\hline 5. & 2015 & Mesquita et al & Portugal & Cross- sectional & Physical & [9] \\
\hline 6. & $2012 b$ & Epp \& Waldner & Canada & Cross- sectional & Physical, psychological, Chemical & [10] \\
\hline 7. & 2012 & Berry et al & United States of America & Cross-sectional & physical & {$[11]$} \\
\hline 8. & 2014 & Shirangi et al & Australia & Cohort & Chemical & [12] \\
\hline 9. & 2008 & Moodley et al & Denmark & Cross- sectional & Biological & [13] \\
\hline 10. & 2009 & Leggat et al & Australia & Cross- sectional & Biological & [14] \\
\hline 11. & 2013 & Paterson et al & UK & Cross- sectional & Biological & [15] \\
\hline 12. & 2010 & Baer et al & USA & Case study & Biological & [16] \\
\hline 13. & 2011 & Boost et al & Hong Kong & Cross-sectional & Biological & [17] \\
\hline 14. & 2017 & Oteo et al & Spain & Cross- sectional & Biological & [18] \\
\hline 15. & 2017 & Teoh et al & Australia & Cross- sectional & Biological & [19] \\
\hline 16. & 2014 & Rivera Benitez et al & Mexico & Cross-sectional & Biological & [20] \\
\hline 17. & 2014 & Verkade et al & Netherlands & Cohort & Biological & [21] \\
\hline 18. & 2014 & Zelenik et al & Slovenia & Case study & Biological & [22] \\
\hline 19. & 2012 & Sayin-Kutlu et al & Turkey & Cross-sectional & Biological & [23] \\
\hline 20. & 2012 & Jackson \& Villaroel & USA & Cross- sectional & Biological & [24] \\
\hline 21. & 2010 & Raso et al & Brazil & Cross-sectional & Biological & {$[25]$} \\
\hline 22. & 2014 & Vest \& Clark & USA & Cross- sectional & Biological & [26] \\
\hline 23. & 2011 & Posthaus et al & Switzerland & Case study & Biological & {$[27]$} \\
\hline 24. & 2016 & Galuppi et al & Italy & Case study & Biological & [28] \\
\hline
\end{tabular}




\begin{tabular}{|c|c|c|c|c|c|c|}
\hline$S / N$ & Year & Authors & Location & Study design & Hazard type & References \\
\hline 25. & 2012 & Rahman et al & Bangladesh & Cross-sectional & Biological & [29] \\
\hline 26. & 2012 & de Rooij et al & Netherlands & Cross- sectional & Biological & [30] \\
\hline 27. & 2013 & Van de Brom et al & Netherlands & Cross-sectional & Biological & [31] \\
\hline 28. & 2015 & Fenga et al & Italy & Cross-sectional & Biological & [32] \\
\hline 29. & 2011 & Archer et al & South Africa & Cross-sectional & Biological & [33] \\
\hline 30. & 2008 & Gait et al & United Kingdom & Case Study & Biological & [34] \\
\hline 31. & 2013 & Moliner et al & Argentina & Cross- sectional & Biological & [35] \\
\hline 32. & 2013 & Ali et al & Pakistan & Cross-sectional & Biological & [36] \\
\hline 33. & 2014 & Lantos et al & USA & Cross- sectional & Biological & [37] \\
\hline
\end{tabular}

more likely to have experienced NSI [9]. It was observed veterinary technicians were significantly $(\mathrm{P}<0.001)$ more likely than veterinarians to report NSI because of their training in recapping needles, a study in the USA reported [5].

Chronic traumatic disorders (CTDs) were also documented among Veterinarians in Canada. Veterinarians experienced severe $(5.2 \%)$ to moderate trauma (52.6\%) in relation to the work environment [10], and was more in females than males [11]. In the same study, one-fourth of the respondents $(1,353)$ reported a CTD during their career, while two-thirds of those reporting CTDs reported chronic or residual problems [11]. Bothmen and women (24.0\% and $28.0 \%$ respectively) documented CTDs requiring treatment or restricting usual occupational activities [11]. Holding instruments (28\%) and standing for surgery (12\%) were perceived reasons for developing CTDs among small animal practioners, while calving manipulations, rectal palpations, and equine dental work were commonly reported perceived causes by large animal veterinarians [11]. Large animal practitioners were found more likely to report CTD than other veterinary practitioners (mixed and small animal), and the injuries were more often around the shoulders, forearms, elbows, hands, or knees [11]. No physical risks were reported for veterinary students in the articles reviewed. Table 2 describes the physical hazards reported among veterinary professionals and associated risk factors.

\subsubsection{Chemical hazards}

Limited chemical hazards and risk factors were identified (Table 2). Exposure to chemicals occured in all veterinary work environment but highest in the private practice $[8,10,12]$. Veterinarians in this category were more likely to be victims of accidental exposure to drugs (hormones, antimicrobials), sterilizing and cleansing agents, gas or injection anaesthesia and pesticides[10]. A gender-exclusive increased risk of birth defects in offsprings occurred more in female veterinarians working in small animal practice following exposure to high dose(s) of pesticides or cytotoxic drugs at least once per week $[8,12]$.

\subsubsection{Specific biological hazards or zoonoses common in the veterinary profession}

Zoonoses naturally transmitted infections between vertebrate animals and man were recognized as major occupational biological risks among the veterinary profession. The most commonly identified zoonoses/ biological risks were methicillin-resistant Staphylococcus aureus infection (MRSA), Q- fever, bartonellosis, cryptosporidiosis, and brucellosis.

Methicillin-resistant Staphylococcus aureus (MRSA): Based on literature, MRSA was identified by various diagnostic methods ranging from the traditional culture to molecular typing techniques [13, 15, 17, 21]. The proportions of MRSA colonization in veterinarians varied from $2.6-14.7 \%$ and veterinarians were six times more at risk of MRSA exposure than non-veterinarians [13]. The direct contact with small animals, cattle or horses predisposed personnel to a higher chance of being MRSA carriers [13]. No exposure was reported among veterinary students.

Coxiella burnetti: This pathogen of occupational importance causes Q-fever in humans. All the four studies included performed serological assays most especially immunofluorescent assays (IFAT) and Enzymelinked immunosorbent assay (ELISA). The prevalence of seropositivity to pathogen ranged from $1.4-73.7 \%$ [4, 
Table 2 A description of all physical and chemical hazards identified in the systematic reviews and the associated risk factors/practices.

\begin{tabular}{|c|c|c|c|c|c|c|}
\hline $\mathrm{S} / \mathrm{N}$ & Year & Authors & Injury type/pathogens & OR/PRR, $95 \% \mathrm{Cl}$, P value & Risk factors or suggested & References \\
\hline 1. & 2009 & Shirangi et al & Birth defects in offspring & $\begin{array}{l}\text { OR: } 5.73,95 \% \mathrm{Cl}: 1.27 \text { - } \\
25.80 \text {, and OR: } 2.39,95 \% \mathrm{Cl}: \\
0.99-5.77 \text {, respectively }\end{array}$ & $\begin{array}{l}\text { High dose of radiation (>10 } \\
\text { x-ray films exposure per } \\
\text { week and exposure to } \\
\text { pesticides at least once } \\
\text { per week }\end{array}$ & [8] \\
\hline 2. & 2014 & Shirangi et al & Birth defects in offspring & $\begin{array}{l}\text { 1. RR: } 2.08,95 \% \mathrm{Cl}: 1.05- \\
\text { 4.15, } \mathrm{P}=0.03 \\
\text { 2. RR: } 2.53,95 \% \mathrm{Cl}: 1.00- \\
\text { 3.48, } \mathrm{P}=0.001 \\
\text { 3. RR: } 3.42,95 \% \mathrm{Cl}: 1.68- \\
\text { 6.92. } \mathrm{P}=0.01\end{array}$ & $\begin{array}{l}\text { 1. Pregnant female } \\
\text { veterinarians handling } \\
\text { cytotoxic drugs daily } \\
2 \text {. Women with unplanned } \\
\text { pregnancies more likely to } \\
\text { handle cytotoxic drugs and } \\
\text { experience increased risk } \\
\text { of birth defects } \\
\text { 3. Working in large animal }\end{array}$ & {$[12]$} \\
\hline 3. & $2012 b$ & Epp \& Waldner & Animal bites and allergies. & $\begin{array}{l}\text { 1. OR: } 18.4,95 \% \mathrm{Cl}: 8.4- \\
\text { 40.2, } \mathrm{P}=0.001 ; \mathrm{OR}: 12.9 \\
95 \% \mathrm{Cl}: 5.8-28.6, \mathrm{P}=0.001 \\
\text { respectively. } \\
\text { 2. OR: } 1.4,95 \% \mathrm{Cl}: 1.1-1.9, \\
\mathrm{p}<0.05\end{array}$ & $\begin{array}{l}\text { 1. Companion animal } \\
\text { veterinarians at higher } \\
\text { risk than food animal } \\
\text { veterinarians; mixed } \\
\text { animal veterinarians than } \\
\text { food animal veterinarians } \\
2 \text {. Female veterinarians } \\
\text { more likely to develop } \\
\text { allergies due to animal } \\
\text { contact than male }\end{array}$ & [10] \\
\hline 4. & 2016 & Fowler et al & $\begin{array}{l}\text { Needlestick and animal- } \\
\text { related injuries. }\end{array}$ & NK & $\begin{array}{l}\text { Common physical hazards } \\
\text { reported }\end{array}$ & {$[5]$} \\
\hline 5. & 2016 & $\begin{array}{l}\text { Mshelbwala } \\
\text { et al }\end{array}$ & Needlestick & $\begin{array}{l}\text { OR: } 2.8,95 \% \mathrm{Cl}: 1.4-6.0, \\
\mathrm{p}=0.006 \text { and } \mathrm{OR}: 2.4, \\
95 \% \mathrm{Cl}: 1.1-6.2, \mathrm{p}=0.036 \\
\text { respectively }\end{array}$ & $\begin{array}{l}\text { Male sex and working } \\
\text { with poultry daily } \\
\text { respectively }\end{array}$ & [7] \\
\hline 6. & 2015 & Mesquita et al & Needlestick & $\begin{array}{l}\text { 1.OR: } 16.54,95 \% \mathrm{Cl} \text { : } \\
\text { 3.69-74.26, } \mathrm{p}<.001 \\
\text { 2. OR: } 145.74,95 \% \mathrm{Cl}: 40.94 \\
-518.78, \mathrm{p}<.001 \\
\text { 3. OR: } 62.73,95 \% \mathrm{Cl}: \\
\text { 7.74-508.4, } \mathrm{p}<.001 \text { and OR: } \\
25.55,95 \% \mathrm{Cl}: 3.75-174.12, \\
\mathrm{p}<.001 \text { respectively }\end{array}$ & $\begin{array}{l}\text { 1. } 11 \text { to } 20 \text { years of } \\
\text { practice more at risk than } 1 \\
\text { to } 10 \text { years. } \\
\text { 2. Worked with dogs } \\
\text { 3. Contact with household } \\
\text { bovine and sheep }\end{array}$ & [9] \\
\hline 7. & $2012 b$ & Epp \& Waldner & $\begin{array}{l}\text { All forms of injury } \\
\text { (needlestick, back } \\
\text { strain, limb strain, fall, } \\
\text { hearing loss, vehicle } \\
\text { accident, assault, head } \\
\text { injury, burns/ frostbite/ } \\
\text { heatstroke, bite/ scratch, } \\
\text { crush/kick/ or trample. } \\
\text { Chemical e.g. accidental } \\
\text { exposures to radiation, } \\
\text { gas anaesthesia, drugs, } \\
\text { pesticides, allergies. } \\
\text { Psychological e.g. stress }\end{array}$ & $\begin{array}{l}\text { 1. OR: } 2.2,95 \% \mathrm{Cl}: 1.5-3.3 \text {; } \\
\text { OR: } 4.5,95 \% \mathrm{Cl}: 1.2-17.1 \\
\text { respectively } \\
\text { 2. OR: } 1.7,95 \% \mathrm{Cl}: 1.2-2.4 \\
\text { 3.OR: } 3.2,95 \% \mathrm{Cl}: 2.2-4.7 ; \\
\text { OR: } 4.9,95 \% \mathrm{Cl}: 3.2-7.5 \\
\text { respectively } \\
\text { 4. } \mathrm{p}=0.005, \mathrm{p}<0.001 \text {, and } \mathrm{p} \\
=0.001 \text { respectively }\end{array}$ & $\begin{array}{l}\text { 1. Working with large } \\
\text { animals, veterinarians who } \\
\text { graduated } 1990-2007 \\
\text { were more likely to be } \\
\text { exposed to at least one } \\
\text { injury than other practices } \\
\text { and who graduated pre- } \\
1990 \text { respectively } \\
\text { 2. Risk of exposures to } \\
\text { pesticides was more likely } \\
\text { in Veterinarians who } \\
\text { graduated pre- } 1990 \\
\text { 3. Accidental exposures to } \\
\text { x-ray and gas anesthetics } \\
\text { were reported more likely } \\
\text { in the pre-1990 graduates. }\end{array}$ & [10] \\
\hline
\end{tabular}




\begin{tabular}{|c|c|c|c|c|c|c|}
\hline$S / N$ & Year & Authors & Injury type/pathogens & OR/PRR, $95 \% \mathrm{Cl}, \mathrm{P}$ value & Risk factors or suggested & References \\
\hline & & & & & $\begin{array}{l}\text { 4. Stress was higher in } \\
\text { post-1990 graduates, } \\
\text { females and those who } \\
\text { worked more than a } \\
\text { 40-hour a week }\end{array}$ & \\
\hline 8. & 2012 & Berry et al & $\begin{array}{l}\text { Cumulative Trauma } \\
\text { Disorders }\end{array}$ & $\begin{array}{l}\text { OR: } 1.72,95 \% \mathrm{Cl}: 1.24-2.39 \text {, } \\
p=0.001 ; \text { OR: } 1.54,95 \% \mathrm{Cl}: \\
1.03-2.32, p=0.037 ; \text { OR: } \\
1.02,95 \% \mathrm{Cl}: 1.01-1.03, p= \\
0.004 ; \text { OR: } \\
1.01,95 \% \mathrm{Cl}: 1.00-1.01, p= \\
0.003 \text { respectively }\end{array}$ & $\begin{array}{l}\text { Risk factors associated } \\
\text { with CTDs in veterinarians } \\
\text { included sex (Female), } \\
\text { working full-time, } \\
\text { rectal palpations, and } \\
\text { large animal practice } \\
\text { respectively. }\end{array}$ & [11] \\
\hline
\end{tabular}

$\mathrm{NK}=$ Not Known; $\mathrm{OR}=$ Odds Ratio; $\mathrm{PRR}=$ Prevalence Risk Ratio; $\mathrm{RR}=$ Relative risk ratio; $\mathrm{Cl}=$ Confidence interval

$24,26,30-32]$. One of the studies recorded $18.7 \%$ among Dutch veterinary students [30]. Risk factors associated with students' exposure based on multivariable logistic regression model were identified to include contact with farm animals, students being in advanced year of study, having had exposure to a zoonosis before the study and having ever lived on a ruminant farm [30]. Alternatively, among the Dutch veterinary professionals, the associated risk of exposure was linked with the hours of contact with animal per week, the number of years of postgraduation, being in the rural or suburban residence, being a practising veterinarian, and occupational contact with swine [31]. (Table 3).

Cryptosporidium Parvum Only two case reports of outbreaks among groups of veterinary students were reported from Italy [28] and the UK [34]. The species were identified in both studies by culturebased methods, PCR-restriction fragment length polymorphism, DNA sequence typing and nested PCR techniques. The outbreak among the Italian veterinary students was plausibly linked with the introduction of Cryptosporidium in an equine perinatology unit (EPU) due to an asymptomatic foal [28], while outbreak among UK students was caused by a 'lapse in hygiene' on a farm with known infected calves [34].

\section{Bartonella species:}

Bartonella species are emerging pathogens in human [37]. High (73.0\%) seroprevalence of infection with Bartonella spp. was found among companion animal veterinary personnel from Spain in one of the studies reviewed [18]. However, the other papers indicated a lower prevalence between 22.0 [23] and 28.0\% [37]. A few risk determinants of exposure to pathogen included being less than 35 years of age [23], contact with ticks [23] and history of travel to Asia [37].

\section{Brucella species:}

The prevalence of infection ranged from $0.1-29.1 \%$ in our review $[29,35,36]$. The pathogen was identified mainly by serology and PCR based techniques. Contact with animals especially goats, number of years of practice or veterinarians working in a zone characterized by a high prevalence of brucellosis were identified as contributing factors for increased exposure to brucellosis (Table 3). The review also showed the more the number of years accumulated as a graduate, the greater the likelihood of illness [35].

\subsection{Meta-analysis of proportion estimates of occupational hazards and ratio measure for working or contact with animals as a risk factor for occupational hazards among veterinarians.}

The pooled proportion estimate of biological hazards among veterinarian was 17\% (95\% CI: 15.0-19.0, $\mathrm{p}<0.001$ ). The overall between-study heterogeneity was significant and substantial $\left(I^{2}=98.98 \%, \mathrm{p}<0.001\right)$. Subgroup analysis of different biological hazards was presented in Figure 3, relatively high proportions 31.0\% (95\% CI: 0.062\%, $\mathrm{P}=0.05$ ), $26.0 \%$ (95\% CI: $16.0-36.0 \%, \mathrm{p}<0.001)$ and $24.0 \%$ (95\% CI: $0.0-49.0 \%, \mathrm{p}<0.05)$ were recorded for bartonellosis, Q-fever and viral infections respectively.

However, the estimated proportion for other biological hazards including brucellosis, leptospirosis, cryptosporidiosis, and MRSA was between $7.0 \%$ and $14.0 \%$ and statistically significant at $\mathrm{p}<0.05$. For the chemical hazard, the pooled proportional estimate was calculated from two studies with 7.0\% (95\% CI: 6.0-9.0\%, P<0.001) estimated proportion (Figure 4). 
Table 3 A summary of biological hazards and the associated risk factors/practices identified for the systematic review

\begin{tabular}{|c|c|c|c|c|c|c|c|}
\hline $\mathrm{S} / \mathrm{N}$ & Year & Authors & $\begin{array}{l}\text { Injury type/ } \\
\text { pathogens }\end{array}$ & $\begin{array}{l}\text { Methods for pathogen } \\
\text { confirmation }\end{array}$ & $\begin{array}{l}\text { OR/PR, } 95 \% \mathrm{Cl}, \mathrm{P} \\
\text { value }\end{array}$ & $\begin{array}{l}\text { Risk factors or } \\
\text { suggested }\end{array}$ & References \\
\hline 1. & $2012 a$ & $\begin{array}{l}\text { Epp \& } \\
\text { Waldner }\end{array}$ & Zoonoses & NK & $\begin{array}{l}\text { OR: } 8.6,95 \% \mathrm{Cl}: 1.1 \\
-65.1, \mathrm{P}=<0.001\end{array}$ & $\begin{array}{l}\text { Mixed animal } \\
\text { veterinarians had } \\
\text { higher odds of } \\
\text { developing a personal } \\
\text { zoonosis than others }\end{array}$ & [4] \\
\hline 2. & 2016 & Fowler et al & $\begin{array}{l}\text { Infectious diseases } \\
\text { (dermatophytosis, } \\
\text { bite wound infection, } \\
\text { salmonellosis, } \\
\text { cryptosporidiosis }\end{array}$ & NK & NK & $\begin{array}{l}\text { Respondents reported } \\
\text { acquiring at least } 1 \\
\text { zoonotic infection at } \\
\text { some point during } \\
\text { their career. }\end{array}$ & [5] \\
\hline 3. & 2008 & $\begin{array}{l}\text { Moodley } \\
\text { et al }\end{array}$ & MRSA & Culture and PCR & $\begin{array}{l}P=0.02, P<0.001, \\
P<0.001 \text { respectively }\end{array}$ & $\begin{array}{l}\text { MRSA exposure } \\
6 \text { times higher in } \\
\text { Veterinarians than } \\
\text { non-veterinarian } \\
\text { professionals, } \\
\text { exposure to small } \\
\text { animals, horses } \\
\text { respectively }\end{array}$ & [13] \\
\hline 4. & 2009 & Leggat et al & Hand dermatitis (HD) & NK & $\begin{array}{l}\text { 1. OR: 4.3, 95\% Cl: } \\
\text { 2.7-6.6, } \mathrm{P}<0.001 \\
\text { 2. OR: } 3.5,95 \% \mathrm{Cl} \text { : } \\
\text { 2.2-5.4, } \mathrm{P}<0.001 \\
\text { 3. OR: } 15.5,95 \% \mathrm{Cl} \text { : } \\
\text { 5.4-44.5, } \\
\mathrm{P}<0.001\end{array}$ & $\begin{array}{l}\text { 1. Veterinarians with } \\
\text { a current allergic } \\
\text { disease } \\
2 . \text { In female } \\
\text { veterinarians } \\
\text { 3. Reporting allergies } \\
\text { within last } 1 \text { year }\end{array}$ & [14] \\
\hline 5. & 2013 & $\begin{array}{l}\text { Paterson } \\
\text { et al }\end{array}$ & $\begin{array}{l}\text { Methicillin-resistant } \\
\text { Staphylococcus } \\
\text { aureus (MRSA) }\end{array}$ & $\begin{array}{l}\text { Culture, multiplex } \\
\text { polymerase chain } \\
\text { reaction (PCR), } \\
\text { matrix assisted laser } \\
\text { desorption/ionization } \\
\text { (MALDI-TOF) }\end{array}$ & NK & Contact with livestock & [15] \\
\hline 6. & 2008 & Baer et al & Leptosira & $\begin{array}{l}\text { Microscopic } \\
\text { agglutination test }\end{array}$ & NK & $\begin{array}{l}\text { Contact with an } \\
\text { apparently healthy } \\
\text { pet rat }\end{array}$ & [16] \\
\hline 7. & 2008 & Boost et al & MRSA & $\begin{array}{l}\text { Culture, } P C R \text {, multilocus } \\
\text { sequence typing (MLST) }\end{array}$ & $\begin{array}{l}\mathrm{P}<0.001, \mathrm{P}=0.03 \\
\text { respectively }\end{array}$ & $\begin{array}{l}\text { Contact with large } \\
\text { animals }\end{array}$ & [17] \\
\hline 8. & 2017 & Oteo et al & Bartonella & $\begin{array}{l}\text { Culture, PCR, DNA } \\
\text { sequencing, }\end{array}$ & NK & $\begin{array}{l}\text { Veterinarians working } \\
\text { with companion } \\
\text { animal }\end{array}$ & [18] \\
\hline 9. & 2017 & Teoh et al & $\begin{array}{l}\text { Rickettsia felis and } R \text {. } \\
\text { typhi }\end{array}$ & $\begin{array}{l}\text { Indirect micro } \\
\text { immunofluorescence } \\
\text { antibody testing } \\
\text { (MIFAT) }\end{array}$ & $\begin{array}{l}\text { OR: } 0.756,95 \% \mathrm{Cl}: \\
\text { 0.582-0.982, P= } \\
\text { 0.04; OR: } 0.752, \\
\text { 95\% Cl: } 0.579- \\
\text { 0.975, P=0.034 } \\
\text { Respectively } \\
\text { 2. OR: } 0.611,95 \% \\
\text { Cl: } 0.38-0.982, \mathrm{P}= \\
0.044 \\
\text { 3. OR: } 1.381,95 \% \\
\text { Cl: } 0.973-1.96, \mathrm{P}= \\
0.075\end{array}$ & $\begin{array}{l}\text { Older veterinarians } \\
\& \text { gt; } 60 \text { years at } \\
\text { reduced risk of } \\
\text { R. felis or generalized } \\
\text { R. felis or R. typhi } \\
\text { 2. Veterinarians } \\
\text { recommending flea } \\
\text { treatments } \\
\text { 3. Veterinarians } \\
\text { located at } \\
\text { southeastern } \\
\text { Australian states of } \\
\text { Victoria or Tasmania }\end{array}$ & [19] \\
\hline
\end{tabular}




\begin{tabular}{|c|c|c|c|c|c|c|c|}
\hline $\mathbf{S} / \mathrm{N}$ & Year & Authors & $\begin{array}{l}\text { Injury type/ } \\
\text { pathogens }\end{array}$ & $\begin{array}{l}\text { Methods for pathogen } \\
\text { confirmation }\end{array}$ & $\begin{array}{l}\mathrm{OR} / \mathrm{PR}, 95 \% \mathrm{Cl}, \mathrm{P} \\
\text { value }\end{array}$ & $\begin{array}{l}\text { Risk factors or } \\
\text { suggested }\end{array}$ & References \\
\hline 10. & 2012 & $\begin{array}{l}\text { Rivera } \\
\text { Benitez et al }\end{array}$ & $\begin{array}{l}\text { Rubula virus, } \\
\text { Encephalomyocarditis } \\
\text { virus \& Leptospira }\end{array}$ & $\begin{array}{l}\text { Hemagglutination } \\
\text { inhibition test, viral } \\
\text { neutralization test }\end{array}$ & OR: $1.38, P<0.05$ & $\begin{array}{l}\text { Number of visits } \\
\text { to farm increased } \\
\text { exposure to } \\
\text { Encephalomyocarditis } \\
\text { virus }\end{array}$ & [20] \\
\hline 11. & 2014 & $\begin{array}{l}\text { Verkade } \\
\text { et al }\end{array}$ & MRSA & $\begin{array}{l}\text { Culture, (spa) typing } \\
\text { and multiple-locus } \\
\text { variable-number } \\
\text { tandem repeat analysis } \\
\text { (MLVA) }\end{array}$ & $\begin{array}{l}\text { PRR: } 9.3 ; 95 \% \mathrm{Cl} 2.8 \\
-38.5 ; \\
\text { PRR: } 2.1 ; 95 \% \mathrm{Cl} 1.0 \\
-4.6 \text { respectively }\end{array}$ & $\begin{array}{l}\text { Veterinarians with } \\
\text { persistent MRSA } \\
\text { significantly increased } \\
\text { carriage in household } \\
\text { members }\end{array}$ & [21] \\
\hline 12. & 2012 & Zelenik et al & Listeria & $\begin{array}{l}\text { Culture, serology, } \\
\text { and pulsed-field gel } \\
\text { electrophoresis (PFGE) }\end{array}$ & NK & $\begin{array}{l}\text { Assisted delivery of a } \\
\text { stillborn calf }\end{array}$ & [22] \\
\hline 13. & 2010 & $\begin{array}{l}\text { Sayin-Kutlu } \\
\text { et al }\end{array}$ & Bartonella & Indirect IFA & $\begin{array}{l}\text { 1.OR: } 5.166 ; 95 \% \\
\mathrm{Cl}, 1.532-17.425, \mathrm{P} \\
=0.008 .\end{array}$ & 1. Age $<35$ years' old & [23] \\
\hline 14. & 2010 & $\begin{array}{l}\text { Jackson \& } \\
\text { Villaroel }\end{array}$ & $\begin{array}{l}\text { Zoonoses - Rabies, } \\
\text { Ringworm, } \\
\text { Sarcoptic mange, } \\
\text { Campylobacteriosis, } \\
\text { Cryptosporidlosis, } \\
\text { Giardiasis }\end{array}$ & NK & $\begin{array}{l}1 . P=0.034 ; P= \\
0.031 \text { respectively }\end{array}$ & $\begin{array}{l}\text { 1. Veterinarians } \\
\text { below } 30 \text { years and } \\
\text { recent graduates } \\
\text { than experienced } \\
\text { veterinarians had } \\
\text { reduced risk }\end{array}$ & [24] \\
\hline 15. & 2008 & Raso et al & Chlamydia psittaci & $\begin{array}{l}\text { Microimmunof- } \\
\text { lurescence (MIF) }\end{array}$ & NK & $\begin{array}{l}\text { Authors suggested } \\
\text { an under-reporting of } \\
\text { this disease in Brazil } \\
\text { and the need for risk } \\
\text { studies }\end{array}$ & [25] \\
\hline 16. & 2012 & Vest \& Clark & Coxiella burnettii & IFAT & $\begin{array}{l}\text { 1. PR: } 1.96 ; 95 \% \\
\mathrm{Cl} 1.15-3.35, \mathrm{P}= \\
0.015 \\
\text { 2. } \mathrm{PR}: 2.16,95 \% \mathrm{Cl} \\
0.91-5.1, \mathrm{P}=0.09 \\
\text { 3. PR: } 2.89,95 \% \\
\mathrm{Cl} 1.13-7.4, \mathrm{P}= \\
0.032, \text { and PR: } 3.17 \text {, } \\
95 \% \mathrm{Cl} 1.03- \\
\text { 9.71, } \mathrm{P}=0.039 \\
\text { respectively }\end{array}$ & $\begin{array}{l}\text { 1. Women } \\
\text { veterinarians than the } \\
\text { men category } \\
2 . \text { Veterinarians within } \\
40-49 \text { age group } \\
3 . \text { Women deployed } \\
\text { to Operation Iraqi } \\
\text { Freedom }\end{array}$ & [26] \\
\hline 17. & 2011 & $\begin{array}{l}\text { Posthaus } \\
\text { et al }\end{array}$ & $\begin{array}{l}\text { Mycobacterium } \\
\text { tuberculosis }\end{array}$ & IFN-g release assay & NK & $\begin{array}{l}\text { This case study } \\
\text { reported the potential } \\
\text { infection in veterinary } \\
\text { personnel with M. } \\
\text { tuberculosis due to } \\
\text { contact infected dog }\end{array}$ & [27] \\
\hline 18. & 2016 & Galuppi et al & $\begin{array}{l}\text { Cryptosporidium } \\
\text { parvum }\end{array}$ & $\begin{array}{l}\text { Ziehl-Neelsen staining, } \\
\text { nested PCR, PCR- } \\
\text { restriction fragment } \\
\text { length polymorphism } \\
\text { analysis (PCR-RFLP) }\end{array}$ & NK & $\begin{array}{l}\text { The case study linked } \\
\text { the transmission of } \\
\text { the pathogen from } \\
\text { foals hospitalized } \\
\text { in an equine } \\
\text { perinatology unit } \\
\text { (EPU) to an outbreak in } \\
\text { veterinary students. }\end{array}$ & [28] \\
\hline
\end{tabular}




\begin{tabular}{|c|c|c|c|c|c|c|c|}
\hline$S / N$ & Year & Authors & $\begin{array}{l}\text { Injury type/ } \\
\text { pathogens }\end{array}$ & $\begin{array}{l}\text { Methods for pathogen } \\
\text { confirmation }\end{array}$ & $\begin{array}{l}\text { OR/PR, } 95 \% \mathrm{Cl}, \mathrm{P} \\
\text { value }\end{array}$ & $\begin{array}{l}\text { Risk factors or } \\
\text { suggested }\end{array}$ & References \\
\hline 19. & 2012 & $\begin{array}{l}\text { Rahman } \\
\text { et al }\end{array}$ & Brucella & $\begin{array}{l}\text { Rose Bengal Test } \\
\text { (RBT), Standard Tube } \\
\text { Agglutination Test } \\
\text { (STAT), iELISA, Q-PCR }\end{array}$ & $\begin{array}{l}\text { 1. OR: } 59.8,95 \% \mathrm{Cl} \text { : } \\
6.40-559.93, p< \\
0.001 \\
\text { 2. OR: } 9.9,95 \% \\
\mathrm{Cl}: 1.03-95.30 \\
\mathrm{p}=0.047 ; \text { OR: } \\
\text { 14.2, } 95 \% \mathrm{Cl}: 1.56 \\
-129.6, p=0.019 \\
\text { respectively }\end{array}$ & $\begin{array}{l}\text { 1. More likely in } \\
\text { handling goats than } \\
\text { cattle. } \\
2 \text {. Duration of contact } \\
\text { with animals for } 16 \text { - } \\
25 \text { and }>26 \text { years than } \\
\leq 5 \text { years respectively }\end{array}$ & [29] \\
\hline 20. & 2012 & de Rooij et al & Coxiella burnettii & $\begin{array}{l}\text { Immunofluorescence } \\
\text { assay (IFA) }\end{array}$ & $\begin{array}{l}\text { OR: } 3.27,95 \% \mathrm{Cl} \\
2.14-5.02 ; \mathrm{OR} \\
\text { year 6: } 2.31,95 \% \\
\mathrm{Cl}: 1.22-4.39 ; \mathrm{OR} \\
\text { year 3-5: } 1.83 \text {, } \\
95 \% \mathrm{Cl}: 1.07- \\
3.10 ; \text { OR: } 1.74, \\
95 \% \mathrm{Cl}: 1.07-2.82 \text {; } \\
\text { OR: } 2.73,95 \% \\
\mathrm{Cl}: 1.59-4.67 \\
\text { respectively }\end{array}$ & $\begin{array}{l}\text { The study direction; } \\
\text { contact with farm } \\
\text { animals; advanced } \\
\text { year of study (years } 6 \\
\text { and 3-5); having had } \\
\text { a zoonosis during the } \\
\text { study, and ever lived } \\
\text { on a ruminant farm } \\
\text { respectively }\end{array}$ & [30] \\
\hline 21. & 2013 & $\begin{array}{l}\text { Van de Brum } \\
\text { et al }\end{array}$ & Coxiella burnettii & $\begin{array}{l}\text { Indirect } \\
\text { immunofluorescent } \\
\text { assay and Enzyme- } \\
\text { linked immunosorbent } \\
\text { assay (ELISA) }\end{array}$ & $\begin{array}{l}\text { 1.OR: 3.9, } 95 \% \\
\mathrm{Cl}: 1.5-10.1, \mathrm{p}= \\
0.005 ; \text { OR: } 13.1 \\
95 \% \mathrm{Cl}: 4.7-36.2, \\
\mathrm{p}<0.001 ; \text { OR: } \\
26.8,95 \% \mathrm{Cl}: 8.1 \\
-88.2, \mathrm{p}<0.001 \\
\text { respectively. }\end{array}$ & $\begin{array}{l}\text { 1. The number of hours } \\
\text { with animal contact } \\
\text { per week that is } 10-19 \\
\text { or } 20-29 \text { or }>=30 \\
\text { hours than< } 10 \text { hours. }\end{array}$ & [31] \\
\hline & & & & & 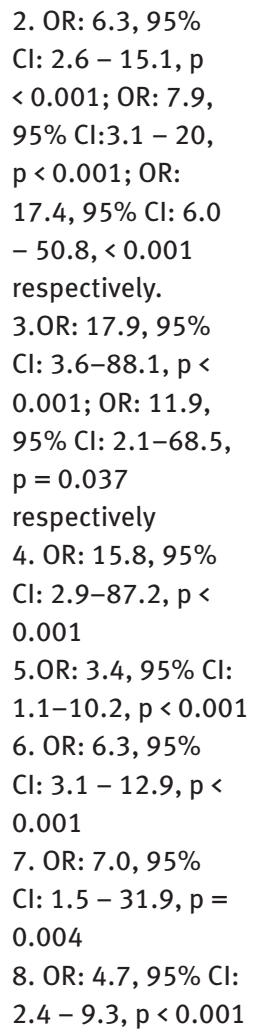 & $\begin{array}{l}\text { 2. Number of years as } \\
\text { veterinarian graduate } \\
\text { i.e. } 3-13 \text { or } 14-21 \\
\text { or }>22 \text { years than } \leq 2 \\
\text { years } \\
\text { 3. Living in a rural or } \\
\text { semi urban area } \\
\text { 4. Livestock } \\
\text { veterinarian } \\
\text { 5. Contact with cows } \\
\text { 6. Contact with } \\
\text { ruminants birth } \\
\text { products } \\
\text { 7. Contact with birth } \\
\text { products of pets } \\
\text { 8. Practicing on cow } \\
\text { farm with reports of } \\
\text { abortion }\end{array}$ & \\
\hline
\end{tabular}




\begin{tabular}{|c|c|c|c|c|c|c|c|}
\hline$S / N$ & Year & Authors & $\begin{array}{l}\text { Injury type/ } \\
\text { pathogens }\end{array}$ & $\begin{array}{l}\text { Methods for pathogen } \\
\text { confirmation }\end{array}$ & $\begin{array}{l}\text { OR/PR, } 95 \% \mathrm{Cl}, \mathrm{P} \\
\text { value }\end{array}$ & $\begin{array}{l}\text { Risk factors or } \\
\text { suggested }\end{array}$ & References \\
\hline 22. & 2015 & Fenga et al & Coxiella burnetii & ELISA & NK & $\begin{array}{l}\text { The study } \\
\text { demonstrated a high } \\
\text { seroprevalence of } C \text {. } \\
\text { burnetii } \\
\text { in animal health } \\
\text { workers including } \\
\text { veterinarians }\end{array}$ & [32] \\
\hline 23. & 2011 & Archer et al & Rift Valley Virus & $\begin{array}{l}\text { ELISA, Real time- PCR } \\
\text { (RT- PCR) and/or loop- } \\
\text { mediated isothermal } \\
\text { amplification assay) } \\
\text { and/or virus isolation }\end{array}$ & $\begin{array}{l}\text { RR: } 16.3,95 \% \mathrm{Cl}: \\
2.3-114.2\end{array}$ & $\begin{array}{l}\text { Performing an animal } \\
\text { autopsy }\end{array}$ & [33] \\
\hline 24. & 2008 & Gait et al & $\begin{array}{l}\text { Cryptosporidium } \\
\text { parvum }\end{array}$ & $\begin{array}{l}\text { (PCR-RFLP) and DNA } \\
\text { sequencing }\end{array}$ & NK & $\begin{array}{l}\text { A case report } \\
\text { of outbreak } \\
\text { cryptosporidiosis } \\
\text { among veterinary } \\
\text { students. The } \\
\text { outbreak was linked to } \\
\text { poor hand hygiene on } \\
\text { a farm with enzootic } C \\
\text { parvum in calves. }\end{array}$ & [34] \\
\hline 25. & 2013 & Moliner et al & $\begin{array}{l}\text { Brucella, } \\
\text { Toxoplasma, } \\
\text { Bacillus anthracis, } \\
\text { Tricophyton, } \\
\text { Leptospira, } \\
\text { Mycobacterium, } \\
\text { others }\end{array}$ & NK & $\begin{array}{l}\text { 1. OR: } 4.79,95 \% \\
\mathrm{Cl}: 2.29-10.01, \\
p=0.0001 ; \text { OR: } \\
8.40,95 \% \mathrm{Cl}: 4.28 \\
-16.48, p=0.0001 \\
\text { respectively } \\
\text { 2. OR: } 2.08,95 \% \\
\mathrm{Cl}: 1.19-3.62, p= \\
0.0099\end{array}$ & $\begin{array}{l}\text { Risk factors identified } \\
\text { for brucellosis were: } \\
1 . \text { The number of } \\
\text { years of } \\
\text { Practice i.e. } 11 \text { - } 20 \\
\text { and }>20 \text { years than } \\
<10 \text { years } \\
2 . \text { Veterinarians } \\
\text { working in a zone } \\
\text { characterized by } \\
\text { a high prevalence } \\
\text { of brucellosis and } \\
\text { specialized agriculture } \\
\text { and milk farms zone }\end{array}$ & [35] \\
\hline 26. & 2013 & Ali et al & Brucella & $\begin{array}{l}\text { RBT, Serum } \\
\text { agglutination test, } \\
\text { RT- PCR }\end{array}$ & $\begin{array}{l}\text { OR: } 1758.5,95 \% \text { Cl: } \\
\text { 48.47- 63799.12, p } \\
<0.001\end{array}$ & $\begin{array}{l}\text { Consumption of } \\
\text { raw milk increased } \\
\text { exposure to } \\
\text { brucellosis }\end{array}$ & [36] \\
\hline 27. & 2014 & Lantos et al & Bartonella & $\begin{array}{l}\text { Culture, PCR, IFA, DNA } \\
\text { sequencing }\end{array}$ & $p=0.006$ & $\begin{array}{l}\text { A history of travel } \\
\text { to Asia was more } \\
\text { common among } \\
\text { veterinary subjects }\end{array}$ & [37] \\
\hline
\end{tabular}

NK = Not Known; $\mathrm{OR}=$ Odds Ratio; $\mathrm{PRR}=$ Prevalence Risk Ratio; $\mathrm{RR}=$ Relative risk ratio; $\mathrm{Cl}$ = Confidence interval

For the physical hazard, a pooled estimate of 65.0\% (95\% CI: 39.0-91.0\%, p<0.001) was calculated. The overall between-study heterogeneity was significant and substantial $\left(I^{2}=99.71 \%, \mathrm{p}<0.001\right)$. The subgroup analysis returned an estimated proportion of $75.0 \%$ (95\% CI: $68.0-82.0 \%, p<0.001)$ for needle stick injury among veterinarians and 25\% (95\% CI: $23.0-27.0 \%, p<0.001)$ for cumulative trauma disorders among the veterinarians (Figure 5).

Meta-analysis was done for only working or contact with animals as a risk factor for occupational hazards among veterinarians (Figure 6). The pooled odds ratio for working 


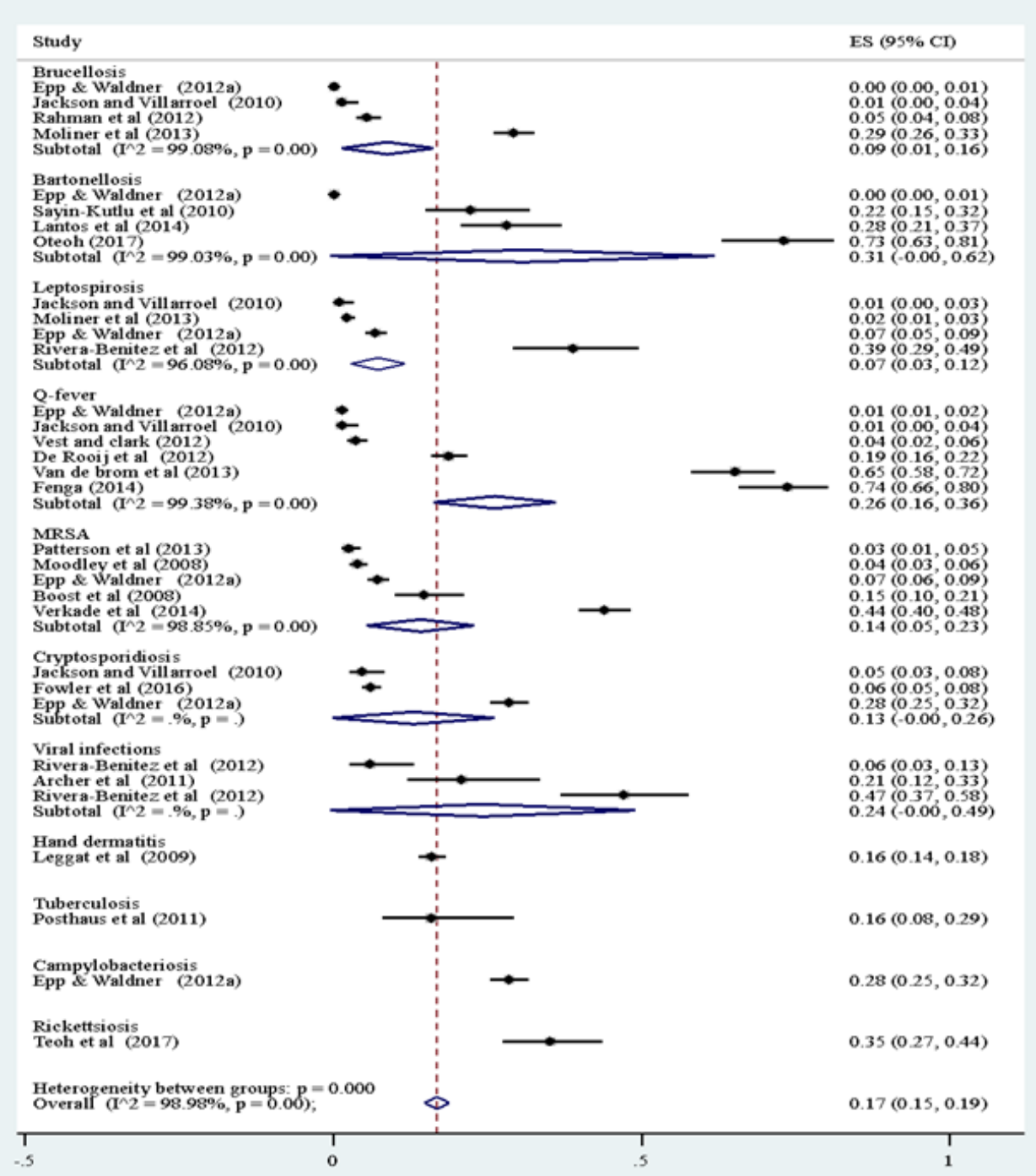

Figure 3 Forest plot of pooled prevalence of biological hazards among veterinarians

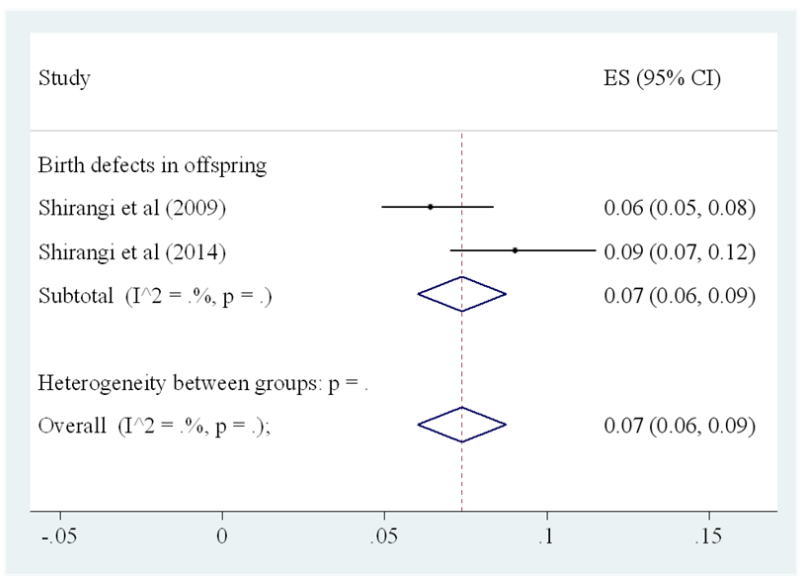

Figure 4 Forest plot of pooled prevalence of chemical hazards among veterinarians

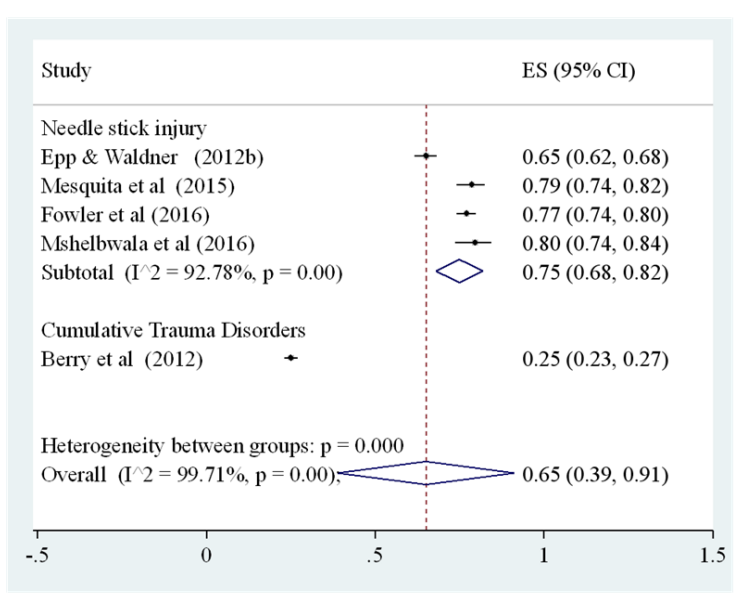

Figure 5 Forest plot of pooled prevalence of physical hazards among veterinarians 


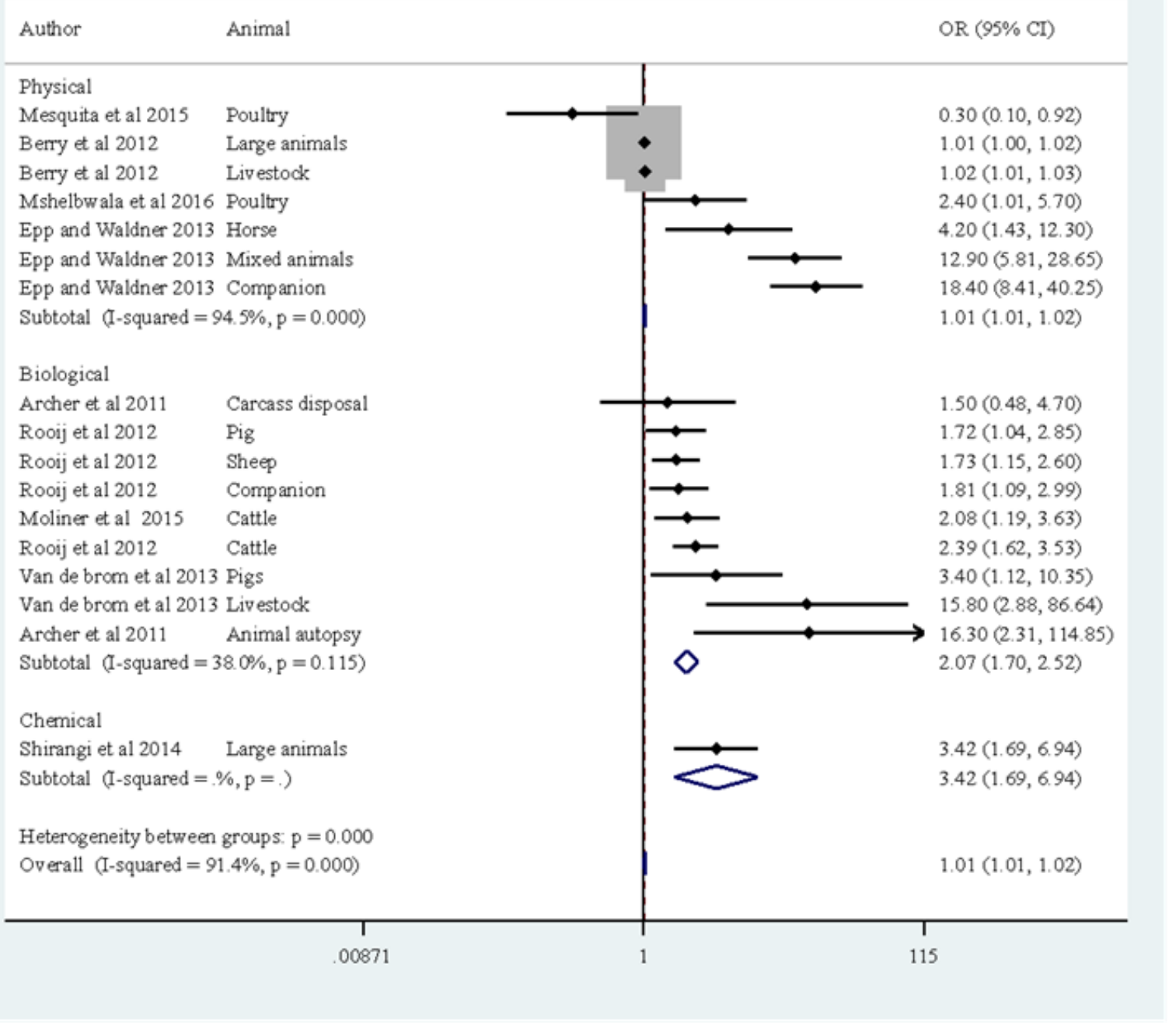

Figure 6 Forest plot of pooled odds ratio estimate of working or contact with animals as a risk factor for occupational hazards among veterinarians.

or contact with animals was $\mathrm{OR}=1.013$ (95\% CI: 1.0081.017, $\mathrm{p}<0.001)$ with overall between-study heterogeneity significant and substantial $\left(I^{2}=99.7 \%, \mathrm{p}<0.001\right)$. Subgroup analysis returned a pooled odds ratio of $\mathrm{OR}=1.012(95 \% \mathrm{CI}$ : 1.008-1.017, $\mathrm{p}<0.001$ ) for working or contact with animals as a risk factor for physical hazards among veterinarian. However, the pooled odds ratio for working or contact with animals as a risk factor for biological hazards among veterinarian was OR=2.07 (95\% CI: 1.70-2.52, p<0.001).

\section{DISCUSSION}

We acknowledge this review may have experienced some limitations based on the number of search database (3) used and the omission of some important studies may have occurred. However, this work has shown that veterinarians and students are at high risk of diverse physical, chemical and biological hazards based on their work profiles contact with animals. The systematic review suggested limited data or documentations on occupational health and safety, particularly risks associated with the veterinary work environment, from the African countries, unlike in the developed ones. Since we began collating data for this study to cover the 10-year period (2007-2017), additional studies in 2020 have been published, most notably on prevalence and risk factors of occupational injuries among veterinary professionals in Ethiopia and India [38,39].

The little documentations on health and safety in Africa may be attributed to weak veterinary services 
and infrastructures, attitudinal issues to occupational health and safety, adherence to and implementation of existing policies, lack of disease/risk surveillance and monitoring systems at national or regional levels, poor laboratory capacitation for early detection and responses, poor risk assessmentsreporting and communication. These factors listed above may be potential contributors to the underestimation of the hazards and may give the false impression that animal health-related workplace occupational hazards are not a problem in Africa. While this review focused on synthesizing and identifying literature on occupational hazards and associated factors in the veterinary setting, it has also revealed a gap for the African continent.

High prevalence of physical hazards, particularly the needlestick injuries (NSIs) and cumulative traumatic disorders (CTDs) among veterinarians are source of concern to the veterinary profession $[4,5,7,9,39,40]$. NSI remains a major hazard in the veterinary profession and mainly associated with recapping of needles and consequent accidental pricks [9,39]. Frequent contact with and handling of large animals such as pigs, goats and sheep increased the risk of NSIs in veterinary students in a study conducted in Nigeria [40]. The lack of in-depth understanding of animal behaviour (ethology), improper handling and disposal of needles, and poor or inadequate restraint techniques applied to these large animals were suggested as potential reasons for the increased risk of NSI [40]. Mitigation measures towards improving training in the best practices relating to the handling, recapping and disposal of needles and sharps are suggested.

Cumulative traumatic disorders (CTDs) in this context are defined as chronic injuries marked with excessive pains in the musculoskeletal system due to overuse or repetitive strain from forceful motions or prolonged uncomfortable positions [41]. In veterinary practices, many activities are physically demanding and have increased potential for injury, especially when dealing with untamed or raging animals [11]. A study showed veterinarians has $\approx 3$ times higher likelihood of CTDs in workplaces when compared with the human doctors [42]. Similarly, large animal veterinarians were more predisposed to CTDs than counterparts in other practice types (small and mixed animal) and increased both in women and men whether they worked full or part-time [11]. Injuries occurred more often in the upper extremities and knees, cases which may become more severe with the pressure of time and work-related stress [11,43]. Large animal practitioners perceived calving manipulations, rectal palpations, and equine dental work most often were the causes of their CTDs, which is understandable because the work is more strenuous dealing with cattle and horses [11]. In another recent study conducted in Ethopia in 2020, a higher prevalence of occupational injuries (OI) was estimated than any developed countries. Also, reported was a significantly higher $\mathrm{OI}$ in government veterinarians than private veterinarians, and exposure to emergency work was a risk factor for injury. However, the use of safety equipment by personnel and history of safety training were found to protect against OIs [38]. On the otherhand, another study from India documented large animal practicing veterinarians faced a higher risk of exposure to injuries, while over half of the veterinarians surveyed reported sustaining work-related injuries due to their contact with animals [39].

Although a few papers were obtained for chemical exposures and risk practices $[8,10,12]$, the pooled estimate proportion was $7.0 \%$ (95\% CI: 6.0-9.0\%). It will appear that chemical hazards and quantitative exposures in the veterinary profession are less studied compared with physical and biological ones. Previous workers have suggested that under-reporting, inconclusive data and the inability to recognize the causal link of hazard were possible reasons for the low prevalence of chemical risks observed in veterinary practices [43]. Veterinarians by practice undertake activities that expose them to chemicals such as pesticides and cytotoxic drugs during prophylaxis or therapy, and anaesthetic gases during preparation for surgeries. Such exposures can lead to lifethreatening illnesses, disorders or complications as grave as cancer [44], reproductive health challenges like birth defects $[8,12]$, disorders of the central nervous system, liver and kidney [44]. The outcome of this study indicated that female veterinarians, most especially those that work in small animal practice were at higher risks of exposure to chemical hazards than their male counterparts [12].

Biological hazards, most particularly zoonoses remained the most documented risks based on the review. Significantly, these diseases still go under-reported in many countries and especially in the developing ones where the risk of co-infections also exists. In this study, almost all of the reported studies originated from the developed economies $(72.7 \%$ originating from Europe, North America and Australia alone). It has been recognized that approximately $61 \%$ of all human pathogens are zoonotic, and $75 \%$ of all emerging pathogens during the past decade have animal source [45]. Therefore, the constant interaction and contact of veterinarians with animals exposes and categorizes them as one of the high-risk groups for zoonoses and or emerging infectious diseases with the consequences of exposure ranging from the simple seroconversion 
to extremely variable symptomatic manifestations or death [43]. The risk is especially problematic to people, such as companion animal owners and veterinary health workers who are immunocompromised [46]. In this work, Zoonoses prevalence ranged from 0.1 to $73.7 \%$ and some common zoonoses identified as threats with relatively high proportions were bartonellosis, Q-fever and other viral infections. Contact with or handling animals increases the risk of exposure to zoonoses by twice in veterinarians (OR=2.07, 95\% CI: 1.70-2.52, $\mathrm{p}<0.001$ ), emphasizing the importance of zoonoses in the veterinary profession. Mitigation strategies to reduce occupational-related zoonotic threats should be addressed through the implementation of policies and legal document that encourages compliance, improved mechanisms for effective risks assessment, communication and surveillance for domestic and wild animals and veterinary population. We encourage more surveys on occupational health, review and documentation of risks and the impact of zoonotic diseases on the veterinary profession, which would contribute to occupational risk prevention [47]. Development of risk assessment plans, health and safety guidelines, best practices, and mitigation systems to reduce workplace-related hazards must be carried out from training institutions to workplace postgraduation [40].

The fact that zero cases of MRSA and physical trauma were reported in veterinary students does not mean this group are less at risk of being colonized by MRSA or acquiring infection. Besides, a study in the Netherland indicated overall MRSA prevalence in veterinary students and doctors involved in farm animal health was about $160 \times$ higher than that among patients at hospital admissions. The study emphasised students and veterinary doctors caring for livestock have a high risk of being colonized by MRSA [48]. Another study underscores the importance of MRSA in veterinary settings and the need for further extensive research to devise contextual control and prevention strategies after reporting the presence of the pandemic and widespread MRSA clones, ST5 and ST59 in veterinary students in Malaysia [49]. Although MRSA was traditionally considered a pathogen causing nosocomial infections, being the so-called HA-MRSA (healthcare-associated methicillin-resistant Staphylococcus aureus), concerns for MRSA has grown due to the different strain types isolated from various animals and the potential to cause human infections. Several reports have suggested the zoonotic potential of MRSA especially from livestock. For instance the first known case of MRSA transmission was reported between cows detected with subclinical mastitis and a person [50]. Another recent study indicated a high zoonotic transmissibility of ST 398 from livestock to especially farm workers and veterinarians [51]. The MRSA strains orginating from livestock (livestock associated - LA) are often divergent from human strains and can be considered zoonosis with veterinarians, cattle farmers and pet owners considered as high risk groups for acquiring infections [51].

Systematic and/or random bias are inherent part of every observational study, this bias can either underestimate or over-estimate the measure of association if not controlled or corrected. To minimize bias in this systematic review and meta-analysis, we conducted rigorous paper quality assessments to ensure papers included in the systematic review and meta- analysis provided adequate methodology, results with reachable conclusions, enough evidence of association between exposure and occupation with $\mathrm{p}$ values and ratios of measure of association. As earlier noted that risk factor meta-analysis was only done for subsample of studies, which documented working or contact with animals as a risk factor for occupational hazards among veterinarians. Bias for the selection of publications were due to the sufficient published articles reporting ratio measures for working or contact with animals compared with other reported risk factors. In addition, this systematic review and meta-analysis was restricted to only observational studies vis-à-vis cohort, case-control and cross-sectional studies. Also, we attempted to minimize systematic bias, variation and heterogeneity between studies included this systematic review and meta-analysis by fitting randomeffect meta-analysis and subgroup analysis. This allows for estimation of group-specific effects and minimizes overestimation of effect as a result of combining studies of different attributes and design.

\section{Conclusions}

This review has provided a better understanding of occupational health and safety status of veterinarians and gaps within the developing countries. Handling or being in contact with animals poses higher exposures to physical and biological hazards. To reduce the risks of exposures to all forms of occupational-related hazards collaborative efforts of all experts in the field is required. Policy formulation and implementation, insurance against work-related hazards, particularly, those at the highest risks of exposure, on-the-job risk appreciation 
training is needed for all category of workers in the veterinary field. Furthermore, more evidence based data on hazard quantification and exposures are needed in the developing countries where little studies have been conducted.

Acknowledgement: We thank the College Librarian, Dr K.A. Owolabi, College of Veterinary Medicine, Federal University of Agriculture Abeokuta, Ogun State, Nigeria for his technical support.

Authors contributions: $\mathrm{OA}$ and $\mathrm{MA}$ conceived and designed this study. OA, OGF and MA conducted the systematic literature review. BA conducted the metaanalyses. OA, OGF, BA and FOF interpreted findings from the review and analyses. OA drafted the manuscript and all other authors revised it critically for important intellectual content. All authors read and approved the final article.

Conflict of interest: Authors state no conflict of interest. Data availability statement: All data generated or analysed during this study are included in this published article.

\section{References}

[1] International Labour Office (ILO). Estimating the economic costs of occupational injuries and illnesses in developing countries: Essential information for Decision-makers. https:// www.ilo.org/wcmsp5/groups/public/---ed_protect/---protrav/--safework/documents/publication/wcms_207690.pdf. Accessed March 29, 2020.

[2] International Labour Office (ILO). Summary of work-related mortality. World Bank division.https://www.ilo.org/moscow/ areas-of-work/occupational-safety-and-health/WCMS_249278/ lang--en/index.htm. Accessed September 9, 2020.

[3] Ryder G. Global cost of work-related injuries and deaths totals almost $\$ 3$ trillion. Safety and Health Magazine. https://www. safetyandhealthmagazine.com/articles/16112-ilo-global-costof-work-related-injuries-and-deaths-totals-almost-3-trillion. Accessed August 31, 2020.

[4] Epp T, Waldner C. Occupational health hazards in veterinary medicine: zoonoses and other biological hazards. Can Vet J. 2012a Feb;53(2):144-50.

[5] Fowler HN, Holzbauer SM, Smith KE, Scheftel JM. Survey of occupational hazards in Minnesota veterinary practices in 2012. J Am Vet Med Assoc. 2016 Jan;248(2):207-18.

[6] Sánchez A, Prats-van der Ham M, Tatay-Dualde J, Paterna A, de la Fe C, Gómez-Martín Á, et al. Zoonoses in Veterinary Students: A Systematic Review of the Literature. PLoS One. 2017 Jan;12(1):e0169534.

[7] Mshelbwala PP, Weese JS, Idris JM. Prevalence of Needlestick Injury and Its Potential Risk among Veterinarians in Nigeria. Vet Med Int. 2016;2016:7639598.
[8] Shirangi A, Fritschi L, Holman CD, Bower C. Birth defects in offspring of female veterinarians. J Occup Environ Med. 2009 May;51(5):525-33.

[9] Mesquita JR, Sousa SI, Vala H, Nascimento MS. The epidemiology of blood-contaminated needlestick injuries among veterinarians in Portugal. J Agromed. 2015;20(2):160-6.

[10] Epp T, Waldner C. Occupational health hazards in veterinary medicine: physical, psychological, and chemical hazards. Can Vet J. 2012b Feb;53(2):151-7.

[11] Berry SL, Susitaival P, Ahmadi A, Schenker MB. Cumulative trauma disorders among California veterinarians. Am J Ind Med. 2012 Sep;55(9):855-61.

[12] Shirangi A, Bower C, Holman CD, Preen DB, Bruce N. A study of handling cytotoxic drugs and risk of birth defects in offspring of female veterinarians. Int J Environ Res Public Health. 2014 Jun;11(6):6216-30.

[13] Moodley A, Nightingale EC, Stegger M, Nielsen SS, Skov RL, Guardabassi L. High risk for nasal carriage of methicillin-resistant Staphylococcus aureus among Danish veterinary practitioners. Scand J Work Environ Health. 2008 Apr;34(2):151-7.

[14] Leggat PA, Smith DR, Speare R. Hand dermatitis among veterinarians from Queensland, Australia. Contact Dermat. 2009 Jun;60(6):336-8.

[15] Paterson GK, Harrison EM, Craven EF, Petersen A, Larsen $A R$, Ellington MJ, et al. Incidence and characterisation of methicillin-resistant Staphylococcus aureus (MRSA) from nasal colonisation in participants attending a cattle veterinary conference in the UK. PLoS One. 2013 Jul;8(7):e68463.

[16] Baer R, Turnberg W, Yu D, Wohrle R. Leptospirosis in a small animal veterinarian: reminder to follow standardized infection control procedures. Zoonoses Public Health. 2010 Jun;57(4):281-4.

[17] Boost MV, So SY, Perreten V. Low rate of methicillin-resistant coagulase-positive staphylococcal colonization of veterinary personnel in Hong Kong. Zoonoses Public Health. 2011 Feb;58(1):36-40.

[18] Oteo JA, Maggi R, Portillo A, Bradley J, García-Álvarez L, San-Martín M, et al. Prevalence of Bartonella spp. by culture, $\mathrm{PCR}$ and serology, in veterinary personnel from Spain. Parasit Vectors. 2017 Nov;10(1):553-61.

[19] Teoh YT, Hii SF, Stevenson MA, Graves S, Rees R, Stenos J, et al. Serological evidence of exposure to Rickettsia felis and Rickettsia typhi in Australian veterinarians. Parasit Vectors. 2017 Mar;10(1):129-36.

[20] Rivera-Benitez JF, Rosas-Estrada K, Pulido-Camarillo E, de la Peña-Moctezuma A, Castillo-Juárez H, Ramírez-Mendoza H. Serological survey of veterinarians to assess the zoonotic potential of three emerging swine diseases in Mexico. Zoonoses Public Health. 2014 Mar;61(2):131-7.

[21] Verkade E, Kluytmans-van den Bergh M, van Benthem B, van Cleef B, van Rijen M, Bosch T, et al. Transmission of methicillinresistant Staphylococcus aureus CC398 from livestock veterinarians to their household members. PLoS One. 2014 Jul;9(7):e100823.

[22] Zelenik K, Avberšek J, Pate M, Lušicky M, Krt B, Ocepek M, et al. Cutaneous listeriosis in a veterinarian with the evidence of zoonotic transmission-a case report. Zoonoses Public Health. 2014 Jun;61(4):238-41.

[23] Sayin-Kutlu S, Ergin C, Kutlu M, Akkaya Y, Akalin S. Bartonella henselae seroprevalence in cattle breeders and veterinarians 
in the rural areas of Aydin and Denizli, Turkey. Zoonoses Public Health. 2012 Sep;59(6):445-9.

[24] Jackson J, Villarroel A. A survey of the risk of zoonoses for veterinarians. Zoonoses Public Health. 2012 May;59(3):193-201.

[25] Raso TF, Carrasco AO, Silva JC, Marvulo MF, Pinto AA. Seroprevalence of antibodies to Chlamydophila psittaci in zoo workers in Brazil. Zoonoses Public Health. 2010 Sep;57(6):411-6.

[26] Vest KG, Clark LL. Serosurvey and observational study of US Army Veterinary Corps officers for $Q$ fever antibodies from 1989 to 2008. Zoonoses Public Health. 2014 Jun;61(4):271-82.

[27] Posthaus H, Bodmer T, Alves L, Oevermann A, Schiller I, Rhodes $\mathrm{SG}$, et al. Accidental infection of veterinary personnel with Mycobacterium tuberculosis at necropsy: a case study. Vet Microbiol. 2011 May;149(3-4):374-80.

[28] Galuppi R, Piva S, Castagnetti C, Sarli G, lacono E, Fioravanti ML, et al. Cryptosporidium parvum: from foal to veterinary students. Vet Parasitol. 2016 Mar;219:53-6. Erratum in: Vet Parasitol. 2016 May; 221:59.

[29] Rahman AK, Dirk B, Fretin D, Saegerman C, Ahmed MU, Muhammad N, et al. Seroprevalence and risk factors for brucellosis in a high-risk group of individuals in Bangladesh. Foodborne Pathog Dis. 2012 Mar;9(3):190-7.

[30] de Rooij MM, Schimmer B, Versteeg B, Schneeberger P, Berends BR, Heederik D, et al. Risk factors of Coxiella burnetii ( $Q$ fever) seropositivity in veterinary medicine students. PLoS One. 2012;7(2):e32108.

[31] Van den Brom R, Schimmer B, Schneeberger PM, Swart WA, van der Hoek W, Vellema P. Seroepidemiological survey for Coxiella burnetii antibodies and associated risk factors in Dutch livestock veterinarians. PLoS One. 2013, 8(1):e54021.

[32] Fenga C, Gangemi S, De Luca A, Calimeri S, Lo Giudice $D$, Pugliese M, et al. Seroprevalence and occupational risk survey for Coxiella burnetii among exposed workers in Sicily, Southern Italy. Int J Occup Med Environ Health. 2015;28(5):901-7.

[33] Archer BN, Weyer J, Paweska J, Nkosi D, Leman P, Tint KS, et al. Outbreak of Rift Valley fever affecting veterinarians and farmers in South Africa, 2008. S Afr Med J. 2011 Apr;101(4):263-6.

[34] Gait R, Soutar RH, Hanson M, Fraser C, Chalmers R. Outbreak of cryptosporidiosis among veterinary students. Vet Rec. 2008 Jun;162(26):843-5.

[35] Molineri A, Signorini ML, Pérez L, Tarabla HD. Zoonoses in rural veterinarians in the central region of Argentina. Aust J Rural Health. 2013 Oct;21(5):285-90.

[36] Ali S, Ali Q, Neubauer H, Melzer F, Elschner M, Khan I, et al. Seroprevalence and risk factors associated with brucellosis as a professional hazard in Pakistan. Foodborne Pathog Dis. 2013 Jun;10(6):500-5.

[37] Lantos PM, Maggi RG, Ferguson B, Varkey J, Park LP, Breitschwerdt EB, et al. Detection of Bartonella species in the blood of veterinarians and veterinary technicians: a newly recognized occupational hazard? Vector Borne Zoonotic Dis. 2014 Aug;14(8):563-70.
[38] Getahun D, Wubshet M, Kebede E, Abate S, Girma E, Ashenefe $B$, et al. Prevalence of occupational injury and associated factors among veterinary professionals in Addis Ababa city, Ethiopia. Ethiop J Public Health Nutr. 2020;2(1):36-40.

[39] Mishra S, Palkhade R. Risk factors and prevalence of work-related injuries and accidents among veterinarians in India. Vet World. 2020 Nov;13(11):2555-64.

[40] Adebowale 00, Afolabi MO, Adesokan HK, Fasanmi OG, Adeyemo OK, Awoyomi OJ, et al. Determinants of Work-Related Risks among Veterinary Clinical Students in South West Nigeria. Vet Med Int. 2020 Aug;2020:2780378.

[41] Bernard BP. Musculoskeletal disorders and workplace factors-A critical review of epidemiologic evidence for work-related musculoskeletal disorders of the neck, upper extremity, and low back. Cincinati, OH, USA: National Institute of Occupational Safety and Health. https://www.cdc.gov/ niosh/docs/97-141/default.html. Assessed June 4,1997.

[42] Nienhaus A, Skudlik C, Seidler A. Work-related accidents and occupational diseases in veterinarians and their staff. Int Arch Occup Environ Health. 2005 Apr;78(3):230-8.

[43] Bonini S, Buonacucina A, Selis L, Peli A, Mutti A, Corradi M. Occupational hazards in veterinarians: an updating. J Vet Sci Technol. 2016;7(3):317.

[44] Fritschi L. Cancer in veterinarians. Occup Environ Med. 2000 May;57(5):289-97.

[45] World Health Organization (WHO). Neglected Zoonotic Diseases. https://www.who.int/neglected_diseases/diseases/ zoonoses/en/. Assessed September 01, 2020.

[46] Kahn LH. Confronting zoonoses, linking human and veterinary medicine. Emerg Infect Dis. 2006 Apr;12(4):556-61.

[47] Sánchez A, Prats-van der Ham M, Tatay-Dualde J, García-Galán A, de la Fe C, Corrales JC, et al. Zoonosis y salud laboral en la profession veterinaria [Zoonosis and occupational health in the veterinary profession]. Rev. Esp. Salud Publica. 2018 Dec;92(3):e1-8.

[48] Wulf M, van Nes A, Eikelenboom-Boskamp A, de Vries J, Melchers W, Klaassen C, et al. Methicillin-resistant Staphylococcus aureus in veterinary doctors and students, the Netherlands. Emerg Infect Dis. 2006 Dec;12(12):1939-41.

[49] Aklilu E, Zunita Z, Hassan L, Cheng CH. Molecular epidemiology of methicillin-resistant Staphylococcus aureus (MRSA) among veterinary students and personnel at a veterinary hospital in Malaysia. Vet Microbiol. 2013 Jun;164(3-4):352-8.

[50] Juhász-Kaszanyitzky E, Jánosi S, Somogyi P, Dán A, van der Graaf-van Bloois L, van Duijkeren E, et al. MRSA transmission between cows and humans. Emerg Infect Dis. 2007 Apr;13(4):630-2.

[51] Pirolo M, Visaggio D, Gioffrè A, Artuso I, Gherardi M, Pavia $G$, et al. Unidirectional animal-to-human transmission of methicillin-resistant Staphylococcus aureus ST398 in pig farming; evidence from a surveillance study in southern Italy. Antimicrob Resist Infect Control. 2019 Nov;8(1):187-96.

[52] Morgan M. Methicillin-resistant Staphylococcus aureus and animals: zoonosis or humanosis? J Antimicrob Chemother. 2008 Dec;62(6):1181-7. 